

\title{
Bivariate Gamma wear processes for track geometry modelling, with application to intervention scheduling
}

Sophie Mercier, Carolina Meier-Hirmer, Michel Roussignol

\section{To cite this version:}

Sophie Mercier, Carolina Meier-Hirmer, Michel Roussignol. Bivariate Gamma wear processes for track geometry modelling, with application to intervention scheduling. Structure and Infrastructure Engineering, 2012, 8 (4), pp.357-366. 10.1080/15732479.2011.563090 . hal-01576989

\section{HAL Id: hal-01576989 \\ https://hal.science/hal-01576989}

Submitted on 24 Aug 2017

HAL is a multi-disciplinary open access archive for the deposit and dissemination of scientific research documents, whether they are published or not. The documents may come from teaching and research institutions in France or abroad, or from public or private research centers.
L'archive ouverte pluridisciplinaire HAL, est destinée au dépôt et à la diffusion de documents scientifiques de niveau recherche, publiés ou non, émanant des établissements d'enseignement et de recherche français ou étrangers, des laboratoires publics ou privés. 


\title{
RESEARCH ARTICLE
}

\section{Bivariate Gamma wear processes for track geometry modelling, with application to intervention scheduling}

\author{
Sophie Mercier ${ }^{1}$, Carolina Meier-Hirmer ${ }^{2}$, Michel Roussignol ${ }^{3}$ \\ ${ }^{1}$ LMAP, Université de Pau et des Pays de l'Adour, France \\ ${ }^{2}$ SNCF, Infrapôle Paris-Saint Lazare, Paris, France \\ ${ }^{3}$ LAMA, Université Paris-Est, Marne-la-Vallée, France \\ (Received 00 Month 200x; final version received 00 Month 200x)
}

This paper discusses the intervention scheduling of a railway track, based on the observation of two dependent randomly increasing deterioration indicators. These two indicators are modeled through a bivariate Gamma process constructed by trivariate reduction. Empirical and maximum likelihood estimators are given for the process parameters and tested on simulated data. An EM algorithm is used to compute the maximum likelihood estimators. A bivariate Gamma process is then fitted to real data of railway track deterioration. Intervention scheduling is defined, ensuring that the railway track remains of good quality with a high probability. The results are compared to those based on both indicators taken separately, and also on one single indicator. The policy based on the joint information is proved to be safer than the other ones, which shows the potential of the bivariate model.

Keywords: Gamma wear process, Lévy process, dependent stochastic processes, reliability, track maintenance.

Corresponding author: sophie.mercier@univ-pau.fr 


\section{Introduction}

This paper is concerned with the intervention scheduling of a railway track, based on the observation of two indicators, which measure the deterioration of the geometry track. The railway track is considered as deteriorated when any of these two indicators is beyond a given threshold. The goal of the paper is the study of the intervention scheduling, which must ensure that, given some observations provided by inspection, the railway track will remain serviceable until the next intervention with a high probability.

The deterioration of the track geometry is characterized by the development of different representative parameters like, for example, the levelling of the track. Figure 1 shows the defects that are measured by two of these parameters: the longitudinal (NL) and transversal (NT) levelling indicators.

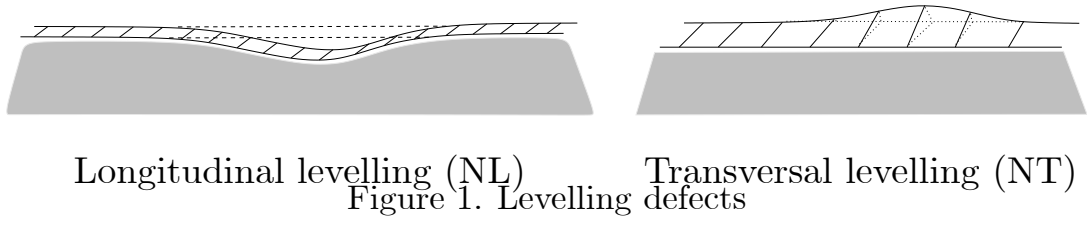

At the SNCF (French National Railways), inspections of the track geometry are carried out by a coach called Mauzin vehicle. On high-speed tracks, a high-speed train (IRIS320) has been adapted to do the same measurements. The collected time series are transformed into indicators that sum up the state of the track over each kilometer. These new indicators are referred to as synthesized Mauzin data. Numeric Mauzin data are available since the opening of the French high-speed lines.

Usually, the synthesized Mauzin indicator of the longitudinal levelling (NL indicator) is used for maintenance issues: thresholds are fixed for this indicator in order to obtain a classification of the track condition and to fix dates for maintenance operations. For example, an intervention should be scheduled before the NL indicator exceeds a predefined threshold.

Based on expert judgements, a Gamma process has been used in (Meier-Hirmer et al. 2009) both to model the evolution of the NL indicator and to plan maintenance actions. As noted by J.M. van Noortwijk in his survey (van Noortwijk 2009), this stochastic process is widely used in reliability studies (see also (Abdel-Hameed 1975, Grall et al. 2002, Zuckerman 1978)). Various domains of applications exist, such as civil engineering 
(Buijs et al. 2005, Cinlar et al. 1977), highway engineering (Nicolai et al. 2007) or railway engineering (Meier-Hirmer et al. 2009). Gamma processes are also used in other domains, such as finance (Joshi and Stacey 2006) or risk analysis (Dufresne et al. 2000). All these papers use univariate Gamma processes.

In the present case, as the two indicators NL and NT are dependent, the use of a bivariate model is required. For this purpose, different processes might be used, such as Bessel (Newby and Baker 2006) or Lévy processes (Kallsen and Tankov 2006). In this paper, the approach of F.A. Buijs, J.W. Hall, J.M. van Noortwijk and P.B. Sayers in (Buijs et al. 2005) is used: a specific Lévy process called bivariate Gamma process is considered. This process is constructed from three independent univariate Gamma processes by trivariate reduction, and has univariate Gamma processes as marginal processes.

It is the first time that both NL and NT indicators are used conjointly to predict the optimal dates of interventions. The objective is to analyze the correlation between the two processes and to determine in what circumstances this bivariate process allows for a better prediction of the intervention times than the current univariate one, based only on the NL indicator.

The paper is organized in the following way: bivariate Gamma processes are introduced in Section 2. Empirical and maximum likelihood estimators for their parameters are provided in Section 3. An EM algorithm is proposed to carry out the maximum likelihood estimation. Both methods are tested on simulated data. Section 4 is devoted to the study of maintenance scheduling and to the comparison of the results based on the bivariate and on the univariate models. Finally, a bivariate Gamma process is fitted to real data of railway track deterioration in Section 5 and it is shown that the maintenance scheduling based on the two available deterioration indicators is clearly safer than those based on a single one, or on both taken separately.

\section{The bivariate Gamma process}

Recall that an univariate (homogeneous) Gamma process $\left(Y_{t}\right)_{t \geq 0}$ with parameters $\alpha>0$ and $\beta>0$ is a process with homogeneous independent increments such that $Y_{t}-Y_{0}$ is Gamma distributed $\Gamma(\alpha t, \beta)$ with probability density function (p.d.f.)

$$
f_{\alpha t, \beta}(x)=\frac{\beta^{\alpha t}}{\Gamma(\alpha t)} x^{\alpha t-1} e^{-\beta x}
$$


for all $x \in[0, \infty)$, and Laplace transform

$$
\mathbb{E}\left(e^{-s\left(Y_{t}-Y_{0}\right)}\right)=\left(\frac{\beta}{\beta+s}\right)^{\alpha t}
$$

for all $s \geq 0$.

Recall that $\mathbb{E}\left(Y_{t}-Y_{0}\right)=\frac{\alpha t}{\beta}, \operatorname{Var}\left(Y_{t}-Y_{0}\right)=\frac{\alpha t}{\beta^{2}}$ for all $t \geq 0$ (see (van Noortwijk 2009) for more details).

Following (Buijs et al. 2005), a bivariate Gamma process $\left(X_{t}\right)_{t \geq 0}=\left(X_{t}^{(1)}, X_{t}^{(2)}\right)_{t \geq 0}$ is constructed by trivariate reduction: starting from three independent univariate Gamma processes $\left(Y_{t}^{(i)}\right)_{t \geq 0}$ with parameters $\left(\alpha_{i}, 1\right)$ for $i \in\{1,2,3\}$ and from $b_{1}>0, b_{2}>0$, one defines:

$$
X_{t}^{(1)}=\left(Y_{t}^{(1)}+Y_{t}^{(3)}\right) / b_{1}, \quad \text { and } \quad X_{t}^{(2)}=\left(Y_{t}^{(2)}+Y_{t}^{(3)}\right) / b_{2} \quad \text { for all } t \geq 0
$$

The process $\left(X_{t}\right)_{t \geq 0}=\left(X_{t}^{(1)}, X_{t}^{(2)}\right)_{t \geq 0}$ is then a homogeneous process in time with independent increments and it is a Lévy process. The marginal processes of $\left(X_{t}\right)_{t \geq 0}$ are univariate Gamma processes with respective parameters $\left(a_{i}, b_{i}\right)$, where $a_{i}=\alpha_{i}+\alpha_{3}$ for $i=1,2$.

For any bivariate Lévy process, the correlation coefficient $\rho_{X_{t}}$ of $X_{t}^{(1)}$ and $X_{t}^{(2)}$ is known to be independent of $t$. For a bivariate Gamma process, one obtains:

$$
\rho=\rho_{X_{t}}=\frac{\alpha_{3}}{\sqrt{a_{1} a_{2}}}
$$

and

$$
\alpha_{1}=a_{1}-\rho \sqrt{a_{1} a_{2}}, \quad \alpha_{2}=a_{2}-\rho \sqrt{a_{1} a_{2}}, \quad \alpha_{3}=\rho \sqrt{a_{1} a_{2}} .
$$

This entails

$$
0 \leq \rho \leq \rho_{\max }=\frac{\min \left(a_{1}, a_{2}\right)}{\sqrt{a_{1} a_{2}}}
$$

See (Devroye 1986) section XI.3 for similar results on bivariate Gamma distributions.

This leads to two equivalent parameterizations of a bivariate Gamma process: $\left(\alpha_{1}, \alpha_{2}, \alpha_{3}, b_{1}, b_{2}\right)$ and $\left(a_{1}, a_{2}, b_{1}, b_{2}, \rho\right)$. 
With the parameterization $\left(\alpha_{1}, \alpha_{2}, \alpha_{3}, b_{1}, b_{2}\right)$, the joint p.d.f. of $X_{t}$ is:

$$
\begin{aligned}
g_{t}\left(x_{1}, x_{2}\right) & =b_{1} b_{2} \int_{0}^{\min \left(b_{1} x_{1}, b_{2} x_{2}\right)} f_{\alpha_{1} t, 1}\left(b_{1} x_{1}-x_{3}\right) f_{\alpha_{2} t, 1}\left(b_{2} x_{2}-x_{3}\right) f_{\alpha_{3} t, 1}\left(x_{3}\right) d x_{3}, \\
& =\frac{b_{1} b_{2} e^{-b_{1} x_{1}-b_{2} x_{2}}}{\Gamma\left(\alpha_{1} t\right) \Gamma\left(\alpha_{2} t\right) \Gamma\left(\alpha_{3} t\right)} \\
& \times \int_{0}^{\min \left(b_{1} x_{1}, b_{2} x_{2}\right)}\left(b_{1} x_{1}-x_{3}\right)^{\alpha_{1} t-1}\left(b_{2} x_{2}-x_{3}\right)^{\alpha_{2} t-1} x_{3}^{\alpha_{3} t-1} e^{-x_{3}} d x_{3}
\end{aligned}
$$

for $x_{1} \geq 0, x_{2} \geq 0$.

A Lévy process is characterized both by its Laplace transform and by its Lévy measure. It is easy to compute their expressions for the bivariate Gamma process constructed above.

For $\mathbf{x}=\left(x_{1}, x_{2}\right)$ with $x_{1} \geq 0$ and $x_{2} \geq 0$, the Laplace transform of $X(t)$ is equal to:

$$
\begin{aligned}
\mathbb{E}\left(e^{-\mathbf{x} \cdot X(t)}\right) & =\mathbb{E}\left(e^{-x_{1} / b_{1} Y_{t}^{(1)}-\left(x_{1} / b_{1}+x_{2} / b_{2}\right) Y_{t}^{(3)}-x_{2} / b_{2} Y_{t}^{(2)}}\right) \\
& =\left(\frac{1}{1+x_{1} / b_{1}}\right)^{\alpha_{1} t}\left(\frac{1}{1+x_{1} / b_{1}+x_{2} / b_{2}}\right)^{\alpha_{3} t}\left(\frac{1}{1+x_{2} / b_{2}}\right)^{\alpha_{2} t} \\
& =\left(\frac{b_{1}}{b_{1}+x_{1}}\right)^{\left(a_{1}-\rho \sqrt{a_{1} a_{2}}\right) t}\left(\frac{b_{1} b_{2}}{b_{1} b_{2}+x_{1} b_{2}+x_{2} b_{1}}\right)^{\rho \sqrt{a_{1} a_{2}} t}\left(\frac{b_{2}}{b_{2}+x_{2}}\right)^{\left(a_{2}-\rho \sqrt{a_{1} a_{2}}\right) t} .
\end{aligned}
$$

Since the Lévy measure $\mu_{X}$ is caracterized by

$$
\mathbb{E}\left(e^{-\mathbf{x} \cdot X_{t}}\right)=\exp \left(t \iint_{[0,+\infty)^{2}}\left(e^{-\mathbf{x} \cdot \mathbf{y}}-1\right) \mu_{X}(d \mathbf{y})\right)
$$

for all $\mathbf{x}=\left(x_{1}, x_{2}\right)$ with $x_{1} \geq 0$ and $x_{2} \geq 0$, it is easy to obtain

$$
\begin{aligned}
d \mu_{X}\left(y_{1}, y_{2}\right) & =\frac{a_{1}-\rho \sqrt{a_{1} a_{2}}}{y_{1}} e^{-b_{1} y_{1}} d y_{1} \times d \delta_{0}\left(y_{2}\right)+\frac{a_{2}-\rho \sqrt{a_{1} a_{2}}}{y_{2}} e^{-b_{2} y_{2}} d \delta_{0}\left(y_{1}\right) \times d y_{2} \\
& +\frac{\rho \sqrt{a_{1} a_{2}}}{y_{1}} e^{-b_{1} y_{1}} d y_{1} \times d \delta_{b_{1} y_{1} / b_{2}}\left(y_{2}\right) .
\end{aligned}
$$

The tail integral function $U_{X}\left(x_{1}, x_{2}\right)=\mu(] x_{1},+\infty[\times] x_{2},+\infty[)$ will also be used in the following. It is equal to:

$$
U\left(x_{1}, x_{2}\right)=\rho \sqrt{a_{1} a_{2}} \int_{\sup \left(b_{1} x_{1}, b_{2} x_{2}\right)}^{+\infty} \frac{e^{-y}}{y} d y .
$$


A more general Gamma process may be constructed as a bivariate Lévy process with univariate Gamma processes as marginal processes. For such a process, if $a_{1}, b_{1}, a_{2}, b_{2}$ are the parameters of the marginal Gamma processes, the linear correlation coefficient $\rho$ may be proved to satisfy:

$$
0 \leq \rho \leq \iint_{[0,+\infty)^{2}} \min \left(\sqrt{\frac{a_{1}}{a_{2}}} E\left(u_{1}\right), \sqrt{\frac{a_{2}}{a_{1}}} E\left(u_{2}\right)\right) d u_{1} d u_{2}
$$

where $E(u)=\int_{u}^{+\infty} \frac{e^{-y}}{y} d y$. If $a_{1} \neq a_{2}$, this upper bound is strictly greater than the upper bound (1) for the process constructed by trivariate reduction. Then the construction by trivariate reduction cannot provide all levels of dependence for Gamma processes since it does not cover the entire range of possible linear correlation coefficients. Nevertheless it is sufficient for our applicative purpose here and in all the following a bivariate Gamma process stands for a process constructed by trivariate reduction.

\section{Parameter estimation}

The data used for the parameter estimation are values of the process increments for non overlapping time intervals on a single trajectory, and also on different independent trajectories. The data can then be represented as realizations of $\left(\Delta t_{j}, \Delta X_{j}^{(1)}, \Delta X_{j}^{(2)}\right)_{1 \leq j \leq n}$ where $\Delta t_{j}=t_{j}-s_{j}$ stands for a time increment and $\Delta X_{j}^{(i)}=X_{t_{j}}^{(i)}-X_{s_{j}}^{(i)}$ for the associated $i$-th marginal increment $(i=1,2)$. For different $j$, the random vectors $\left(\Delta X_{j}^{(1)}, \Delta X_{j}^{(2)}\right)$ are independent, but not identically distributed. The random variable $\Delta X_{j}^{(i)}(i=1,2)$ is Gamma distributed with parameters $\left(a_{i} \Delta t_{j}, b_{i}\right)$. The joint p.d.f. of the random vector $\left(\Delta X_{j}^{(1)}, \Delta X_{j}^{(2)}\right)$ is equal to $g_{\Delta t_{j}}(.,$.$) , with \Delta t_{j}$ substituted to $t$ in (2). In the same way as for the parameter estimation of an univariate Gamma process, both empirical and maximum likelihood methods are possible in the bivariate case.

\subsection{Empirical estimators}

Using $\mathbb{E}\left(\Delta X_{j}^{(i)}\right)=\frac{a_{i}}{b_{i}} \Delta t_{j}$ and $\operatorname{Var}\left(\Delta X_{j}^{(i)}\right)=\frac{a_{i}}{b_{i}^{2}} \Delta t_{j}$ for $i=1,2$ and for all $j$, empirical estimators $\left(\hat{a}_{1}, \hat{b}_{1}, \hat{a}_{2}, \hat{b}_{2}\right)$ of $\left(a_{1}, b_{1}, a_{2}, b_{2}\right)$ are given in (Cinlar et al. 1977) and (van 
Noortwijk and Pandey 2004), with:

$$
\frac{\hat{a}_{i}}{\hat{b}_{i}}=\frac{\sum_{j=1}^{n} \Delta X_{j}^{(i)}}{\sum_{j=1}^{n} \Delta t_{j}} \quad \text { and } \quad \frac{\hat{a}_{i}}{\hat{b}_{i}^{2}}=\frac{\sum_{j=1}^{n}\left(\Delta X_{j}^{(i)}-\frac{\hat{a}_{i}}{\hat{b}_{i}} \Delta t_{j}\right)^{2}}{\sum_{j=1}^{n} \Delta t_{j}-\frac{1}{\sum_{j=1}^{n} \Delta t_{j}} \sum_{j=1}^{n}\left(\Delta t_{j}\right)^{2}}
$$

Using

$$
\operatorname{Cov}\left(\Delta X_{j}^{(1)}, \Delta X_{j}^{(2)}\right)=\rho \frac{\sqrt{a_{1} a_{2}}}{b_{1} b_{2}} \Delta t_{j}
$$

a similar estimator $\hat{\rho}$ may be given for $\rho$, with:

$$
\hat{\rho} \frac{\sqrt{\hat{a}_{1} \hat{a}_{2}}}{\hat{b}_{1} \hat{b}_{2}}=\frac{\sum_{j=1}^{n}\left(\Delta X_{j}^{(1)}-\frac{\hat{a}_{1}}{\hat{b}_{1}} \Delta t_{j}\right)\left(\Delta X_{j}^{(2)}-\frac{\hat{a}_{2}}{\hat{b}_{2}} \Delta t_{j}\right)}{\sum_{j=1}^{n} \Delta t_{j}-\frac{1}{\sum_{j=1}^{n} \Delta t_{j}} \sum_{j=1}^{n}\left(\Delta t_{j}\right)^{2}} .
$$

These estimators satisfy:

$$
\mathbb{E}\left(\frac{\hat{a}_{i}}{\hat{b}_{i}}\right)=\frac{a_{i}}{b_{i}}, \quad \mathbb{E}\left(\frac{\hat{a}_{i}}{\hat{b}_{i}^{2}}\right)=\frac{a_{i}}{b_{i}^{2}}, \quad \mathbb{E}\left(\hat{\rho} \frac{\sqrt{\hat{a}_{1} \hat{a}_{2}}}{\hat{b}_{1} \hat{b}_{2}}\right)=\rho \frac{\sqrt{a_{1} a_{2}}}{b_{1} b_{2}} .
$$

If the time increments $\Delta t_{j}$ are equal, these estimators coïncide with the usual empirical estimators in the case of i.i.d. random variables.

\subsection{Maximum likelihood estimators}

The parameter estimation of a univariate Gamma process is usually done by maximizing the likelihood function (see e.g. (Meier-Hirmer et al. 2009)). With this method, estimators $\bar{a}_{i}$ and $\bar{b}_{i}(i=1,2)$ of the marginal parameters are computed by solving the equations:

$$
\begin{gathered}
\bar{a}_{i}=\frac{\sum_{j=1}^{n} \Delta X_{j}^{(i)}}{\bar{b}_{i}}=\frac{\text { and }}{\sum_{j=1}^{n} \Delta t_{j}} \\
\left(\sum_{j=1}^{n} \Delta t_{j}\right) \times \ln \left(\bar{a}_{i} \frac{\sum_{j=1}^{n} \Delta t_{j}}{\sum_{j=1}^{n} \Delta X_{j}^{(i)}}\right)+\sum_{j=1}^{n} \Delta t_{j}\left(\ln \left(\Delta X_{j}^{(i)}\right)-\psi\left(\bar{a}_{i} \Delta t_{j}\right)\right)=0,
\end{gathered}
$$

where

$$
\psi(x)=\frac{\frac{\mathrm{d} \Gamma(x)}{\mathrm{d} x}}{\Gamma(x)}, \quad \Gamma(x)=\int_{0}^{\infty} e^{-u} u^{x-1} d u
$$

for all $x>0$ ( $\psi$ is the Digamma function). 
In order to estimate all the parameters of the bivariate process $\left(\alpha_{1}, \alpha_{2}, \alpha_{3}, b_{1}, b_{2}\right)$ (which are here preferred to $\left.\left(a_{1}, b_{1}, a_{2}, b_{2}, \rho\right)\right)$, the likelihood function associated with the data $\left(\Delta t_{j}, \Delta X_{j}^{(1)}, \Delta X_{j}^{(2)}\right)_{1 \leq j \leq n}$ can be written as $\mathcal{L}\left(\alpha_{1}, \alpha_{2}, \alpha_{3}, b_{1}, b_{2}\right)=\prod_{j=1}^{n} g_{\Delta t_{j}}\left(\Delta X_{j}^{(1)}, \Delta X_{j}^{(2)}\right)$ where $g_{\Delta t_{j}}$ is provided by (2). However, because of the expression of the function $g_{t}(.,$.$) , it seems complicated to optimize this$ likelihood function directly. An EM algorithm (see (Dempster et al. 1977)) is then used, considering $\left(\Delta Y_{j}^{(3)}=Y_{t_{j}}^{(3)}-Y_{s_{j}}^{(3)}\right)_{1 \leq j \leq n}$ as hidden data. This procedure is still too complicated for estimating the five parameters and does not work numerically. So, the procedure is restricted to the three parameters $\left(\alpha_{1}, \alpha_{2}, \alpha_{3}\right)$. For the parameters $b_{1}, b_{2}$, the values $\left(\bar{b}_{1}, \bar{b}_{2}\right)$ computed using the maximum likelihood method for each univariate marginal process are taken.

For sake of simplicity, the realizations of $\left(\Delta t_{j}, \Delta X_{j}^{(1)}, \Delta X_{j}^{(2)}, \Delta Y_{j}^{(3)}\right)_{1 \leq j \leq n}$ are denoted by $\left(t_{j}, x_{j}^{(1)}, x_{j}^{(2)}, y_{j}^{(3)}\right)_{1<j \leq n}$ in the following, the associated $n$-dimensional random vectors by $\left(\bar{X}^{(1)}, \bar{X}^{(2)}, \bar{Y}^{(3)}\right)$ and the associated $n$-dimensional data vectors by $\left(\bar{x}^{(1)}, \bar{x}^{(2)}, \bar{y}^{(3)}\right)$.

The joint p.d.f. of the random vector $\left(X_{t}^{(1)}, X_{t}^{(2)}, Y_{t}^{(3)}\right)$ is equal to:

$$
\begin{aligned}
& b_{1} b_{2} f_{\alpha_{1} t, 1}\left(b_{1} x_{1}-y_{3}\right) f_{\alpha_{2} t, 1}\left(b_{2} x_{2}-y_{3}\right) f_{\alpha_{3} t, 1}\left(y_{3}\right) \\
& =\frac{b_{1} b_{2}}{\Gamma\left(\alpha_{1} t\right) \Gamma\left(\alpha_{2} t\right) \Gamma\left(\alpha_{3} t\right)} e^{-\left(b_{1} x_{1}+b_{2} x_{2}\right)}\left(b_{1} x_{1}-y_{3}\right)^{\alpha_{1} t-1}\left(b_{2} x_{2}-y_{3}\right)^{\alpha_{2} t-1} y_{3}^{\alpha_{3} t-1} e^{y_{3}}
\end{aligned}
$$

with $0 \leq y_{3} \leq \min \left(b_{1} x_{1}, b_{2} x_{2}\right), x_{1}>0$ and $x_{2}>0$.

Then, the log-likelihood function $Q\left(\bar{x}^{(1)}, \bar{x}^{(2)}, \bar{y}^{(3)}\right)$ associated with the complete data $\left(\bar{x}^{(1)}, \bar{x}^{(2)}, \bar{y}^{(3)}\right)$ is derived:

$$
\begin{aligned}
& Q\left(\bar{x}^{(1)}, \bar{x}^{(2)}, \bar{y}^{(3)}\right) \\
& =n\left(\ln \left(b_{1}\right)+\ln \left(b_{2}\right)\right)-\sum_{j=1}^{n}\left(\ln \Gamma\left(\alpha_{1} t_{j}\right)+\ln \Gamma\left(\alpha_{2} t_{j}\right)+\ln \Gamma\left(\alpha_{3} t_{j}\right)\right)-b_{1} \sum_{j=1}^{n} x_{j}^{(1)} \\
& -b_{2} \sum_{j=1}^{n} x_{j}^{(2)}+\sum_{j=1}^{n}\left(\left(\alpha_{1} t_{j}-1\right) \ln \left(b_{1} x_{j}^{(1)}-y_{j}^{(3)}\right)+\left(\alpha_{2} t_{j}-1\right) \ln \left(b_{2} x_{j}^{(2)}-y_{j}^{(3)}\right)\right. \\
& \left.+\left(\alpha_{3} t_{j}-1\right) \ln \left(y_{j}^{(3)}\right)+y_{j}^{(3)}\right) .
\end{aligned}
$$

For the EM algorithm, the conditional log-likelihood of the complete data given the 
observed data is needed:

$$
\begin{aligned}
& \mathbb{E}\left(Q\left(\bar{X}^{(1)}, \bar{X}^{(2)}, \bar{Y}^{(3)}\right) \mid \bar{X}^{(1)}=\bar{x}^{(1)}, \bar{X}^{(2)}=\bar{x}^{(2)}\right) \\
& =n\left(\ln \left(b_{1}\right)+\ln \left(b_{2}\right)\right)-b_{1} \sum_{j=1}^{n} x_{j}^{(1)}-b_{2} \sum_{j=1}^{n} x_{j}^{(2)} \\
& +\sum_{j=1}^{n}\left(\left(\alpha_{1} t_{j}-1\right) \mathbb{E}\left(\ln \left(b_{1} x_{j}^{(1)}-\Delta Y_{j}^{(3)}\right) \mid \Delta X_{j}^{(1)}=x_{j}^{(1)}, \Delta X_{j}^{(2)}=x_{j}^{(2)}\right)\right. \\
& +\left(\alpha_{2} t_{j}-1\right) \mathbb{E}\left(\ln \left(b_{2} x_{j}^{(2)}-\Delta Y_{j}^{(3)}\right) \mid \Delta X_{j}^{(1)}=x_{j}^{(1)}, \Delta X_{j}^{(2)}=x_{j}^{(2)}\right) \\
& +\left(\alpha_{3} t_{j}-1\right) \mathbb{E}\left(\ln \left(\Delta Y_{j}^{(3)}\right) \mid \Delta X_{j}^{(1)}=x_{j}^{(1)}, \Delta X_{j}^{(2)}=x_{j}^{(2)}\right) \\
& \left.\quad+\mathbb{E}\left(Y_{j}^{(3)} \mid \Delta X_{j}^{(1)}=x_{j}^{(1)}, \Delta X_{j}^{(2)}=x_{j}^{(2)}\right)\right) \\
& -\sum_{j=1}^{n}\left(\ln \Gamma\left(\alpha_{1} t_{j}\right)+\ln \Gamma\left(\alpha_{2} t_{j}\right)+\ln \Gamma\left(\alpha_{3} t_{j}\right)\right) .
\end{aligned}
$$

Finally, the conditional probability density function of $Y_{t}^{(3)}$ given $X_{t}^{(1)}=x_{1}, X_{t}^{(2)}=x_{2}$ is equal to:

$$
\begin{aligned}
& \frac{f_{\alpha_{1} t, 1}\left(b_{1} x_{1}-y_{3}\right) f_{\alpha_{2} t, 1}\left(b_{2} x_{2}-y_{3}\right) f_{\alpha_{3} t, 1}\left(y_{3}\right)}{\int_{0}^{\min \left(b_{1} x_{1}, b_{2} x_{2}\right)} f_{\alpha_{1} t, 1}\left(b_{1} x_{1}-x_{3}\right) f_{\alpha_{2} t, 1}\left(b_{2} x_{2}-x_{3}\right) f_{\alpha_{3} t, 1}\left(x_{3}\right) d x_{3}} \\
& =\frac{\left(b_{1} x_{1}-y_{3}\right)^{\alpha_{1} t-1}\left(b_{2} x_{2}-y_{3}\right)^{\alpha_{2} t-1} y_{3}^{\alpha_{3} t-1} e^{y_{3}}}{\int_{0}^{\min \left(b_{1} x_{1}, b_{2} x_{2}\right)}\left(b_{1} x_{1}-x_{3}\right)^{\alpha_{1} t-1}\left(b_{2} x_{2}-x_{3}\right)^{\alpha_{2} t-1} x_{3}^{\alpha_{3} t-1} e^{x_{3}} d x_{3}}
\end{aligned}
$$

where $0 \leq y_{3} \leq \min \left(b_{1} x_{1}, b_{2} x_{2}\right), x_{1}>0$ and $x_{2}>0$.

Step $k$ of the EM algorithm consists of computing new parameter values $\left(\alpha_{1}^{(k+1)}, \alpha_{2}^{(k+1)}, \alpha_{3}^{(k+1)}\right)$ given the current values $\left(\alpha_{1}^{(k)}, \alpha_{2}^{(k)}, \alpha_{3}^{(k)}\right)$ in two stages:

- stage 1: compute the conditional expectations in (6) using the current set $\left(\alpha_{1}^{(k)}, \alpha_{2}^{(k)}, \alpha_{3}^{(k)}\right)$ of parameters, with:

$$
\begin{aligned}
f_{1}\left(j, \alpha_{1}^{k)}, \alpha_{2}^{(k)}, \alpha_{3}^{(k)}\right) & =\mathbb{E}\left(\ln \left(\bar{b}_{1} \bar{x}_{j}^{(1)}-\bar{Y}_{j}^{(3)}\right) \mid \bar{X}^{(1)}=\bar{x}_{j}^{(1)}, \bar{X}^{(2)}=\bar{x}_{j}^{(2)}\right), \\
f_{2}\left(j, \alpha_{1}^{k)}, \alpha_{2}^{(k)}, \alpha_{3}^{(k)}\right) & =\mathbb{E}\left(\ln \left(\bar{b}_{2} \bar{x}_{j}^{(2)}-\bar{Y}_{j}^{(3)}\right) \mid \bar{X}^{(1)}=\bar{x}_{j}^{(1)}, \bar{X}^{(2)}=\bar{x}_{j}^{(2)}\right), \\
f_{3}\left(j, \alpha_{1}^{k)}, \alpha_{2}^{(k)}, \alpha_{3}^{(k)}\right) & =\mathbb{E}\left(\ln \left(\bar{Y}_{j}^{(3)}\right) \mid \bar{X}^{(1)}=\bar{x}_{j}^{(1)}, \bar{X}^{(2)}=\bar{x}_{j}^{(2)}\right), \\
h\left(\alpha_{1}^{k)}, \alpha_{2}^{(k)}, \alpha_{3}^{(k)}\right) & =\sum_{j=1}^{n} \mathbb{E}\left(\bar{Y}_{j}^{(3)} \mid \bar{X}^{(1)}=\bar{x}_{j}^{(1)}, \bar{X}^{(2)}=\bar{x}_{j}^{(2)}\right) .
\end{aligned}
$$

- stage 2: take for $\left(\alpha_{1}^{(k+1)}, \alpha_{2}^{(k+1)}, \alpha_{3}^{(k+1)}\right)$ the values of $\left(\alpha_{1}, \alpha_{2}, \alpha_{3}\right)$ that maximize $(6)$, 
which here becomes:

$$
\begin{aligned}
& g\left(\alpha_{1}, \alpha_{2}, \alpha_{3}, \alpha_{1}^{(k)}, \alpha_{2}^{(k)}, \alpha_{3}^{(k)}\right) \\
& =n\left(\ln \left(\bar{b}_{1}\right)+\ln \left(\bar{b}_{2}\right)\right)-\bar{b}_{1} \sum_{j=1}^{n} x_{j}^{(1)}-\bar{b}_{2} \sum_{j=1}^{n} x_{j}^{(2)} \\
& +\sum_{j=1}^{n}\left(\left(\alpha_{1} t_{j}-1\right) f_{1}\left(j, \alpha_{1}^{(k)}, \alpha_{2}^{(k)}, \alpha_{3}^{(k)}\right)+\left(\alpha_{2} t_{j}-1\right) f_{2}\left(j, \alpha_{1}^{(k)}, \alpha_{2}^{(k)}, \alpha_{3}^{(k)}\right)\right. \\
& \left.+\left(\alpha_{3} t_{j}-1\right) f_{3}\left(j, \alpha_{1}^{(k)}, \alpha_{2}^{(k)}, \alpha_{3}^{(k)}\right)\right) \\
& -\sum_{j=1}^{n}\left(\ln \Gamma\left(\alpha_{1} t_{j}\right)+\ln \Gamma\left(\alpha_{2} t_{j}\right)+\ln \Gamma\left(\alpha_{3} t_{j}\right)\right)+h\left(\alpha_{1}^{(k)}, \alpha_{2}^{(k)}, \alpha_{3}^{(k)}\right) .
\end{aligned}
$$

The maximization procedure in stage 2 is done by solving the following equation with respect to $\alpha_{i}$ :

$$
\frac{\partial g\left(\alpha_{1}, \alpha_{2}, \alpha_{3}, \alpha_{1}^{(k)}, \alpha_{2}^{(k)}, \alpha_{3}^{(k)}\right)}{\partial \alpha_{i}}=\sum_{j=1}^{n} t_{j} f_{i}\left(j, \alpha_{1}^{(k)}, \alpha_{2}^{(k)}, \alpha_{3}^{(k)}\right)-\sum_{j=1}^{n} t_{j} \psi\left(\alpha_{i} t_{j}\right)=0
$$

for $i=1,2,3$.

This EM algorithm provides estimates for $\alpha_{1}, \alpha_{2}, \alpha_{3}$ using the univariate ML estimates $\left(\bar{b}_{1}, \bar{b}_{2}\right)$ and consequently delivers estimates for $a_{1}, a_{2}$ and $\rho$.

In the same way, it is possible to estimate only $\alpha_{3}$ by an EM algorithm using the univariate ML estimates $\left(\bar{a}_{1}, \bar{b}_{1}, \bar{a}_{2}, \bar{b}_{2}\right)$. In that case, $\alpha_{3}^{(k+1)}$ is the solution of the equation:

$$
\begin{aligned}
& \sum_{j=1}^{n} t_{j}\left(f_{3}\left(j, \alpha_{1}^{(k)}, \alpha_{2}^{(k)}, \alpha_{3}^{(k)}\right)-f_{1}\left(j, \alpha_{1}^{(k)}, \alpha_{2}^{(k)}, \alpha_{3}^{(k)}\right)-f_{2}\left(j, \alpha_{1}^{(k)}, \alpha_{2}^{(k)}, \alpha_{3}^{(k)}\right)\right) \\
& -\sum_{j=1}^{n} t_{j}\left(\psi\left(\alpha_{3} t_{j}\right)-\psi\left(\left(\bar{a}_{1}-\alpha_{3}\right) t_{j}\right)-\psi\left(\left(\bar{a}_{2}-\alpha_{3}\right) t_{j}\right)\right)=0 .
\end{aligned}
$$

\subsection{Tests on simulated data}

We now test the previous methods on simulated data. 500 time increments $\left(t_{j}\right)_{1 \leq j \leq 500}$ are randomly chosen with similar magnitude as the data of track deterioration (the proposed methods will be used on these data in Section 5). Then, 500 values of a bivariate Gamma process with parameters $a_{1}=0.33, a_{2}=0.035, b_{1}=13.5, b_{2}=20$ and $\rho=0.5296$ are simulated corresponding to these time increments. Here again the parameter values have the same order of magnitude than those observed for track deterioration studied 
in Section 5. Three series of 500 data points are simulated independently. Results of parameters estimation are given in Tables 1, 2 and 3, each corresponding to independent series of data. In these tables, one can find: the true values in column 2 , the empirical estimators in column 3 , the univariate maximum likelihood estimators of $a_{1}, b_{1}, a_{2}, b_{2}$ in column 4 , the EM estimator of the three parameters $a_{1}, a_{2}, \rho$ in column 5 , using the parameters $\bar{b}_{1}, \bar{b}_{2}$ previously estimated by the univariate maximum likelihood method (from column 4), and the second EM estimator of the parameter $\rho$ in column 6 , using the estimated parameters $\bar{a}_{1}, \bar{b}_{1}, \bar{a}_{2}, \bar{b}_{2}$ from column 4 .

The initial values for the EM algorithm are different for the three tables. For Table 1, the EM algorithm has been initiated with $\alpha_{1}^{(0)}=\alpha_{2}^{(0)}=0.05$ and $\alpha_{3}^{(0)}=0.15$ (which corresponds to $a_{1}^{(0)}=a_{2}^{(0)}=0.1$ and $\left.\rho^{(0)}=0.75\right)$. For Tables 2 and $3, \alpha_{1}^{(0)}=\alpha_{2}^{(0)}=$ $\alpha_{3}^{(0)}=0.01$, and $\alpha_{1}^{(0)}=0.02, \alpha_{2}^{(0)}=0.01, \alpha_{3}^{(0)}=0.05$ were taken respectively.

\begin{tabular}{|c|c|c|c|c|c|}
\hline & True & Empirical & Univariate & \multicolumn{2}{|c|}{ EM algorithm } \\
& values & estimators & max likelihood & EM1 & EM2 \\
\hline$a_{1}$ & 0.0330 & 0.0348 & 0.0342 & 0.0347 & - \\
$b_{1}$ & 13.5 & 14.38 & 14.14 & - & - \\
$a_{2}$ & 0.0350 & 0.0362 & 0.0357 & 0.0354 & - \\
$b_{2}$ & 20 & 20.58 & 20.25 & - & - \\
$\rho$ & 0.5296 & 0.5637 & - & 0.5231 & 0.5214 \\
\hline
\end{tabular}

Table 1 : Results for the first series of data.

\begin{tabular}{|c|c|c|c|c|c|}
\hline & True & Empirical & Univariate & \multicolumn{2}{|c|}{ EM algorithm } \\
& values & estimators & max likelihood & EM1 & EM2 \\
\hline$a_{1}$ & 0.0330 & 0.0315 & 0.0326 & 0.0328 & - \\
$b_{1}$ & 13.5 & 12.80 & 13.16 & - & - \\
$a_{2}$ & 0.0350 & 0.0357 & 0.0361 & 0.0365 & - \\
$b_{2}$ & 20 & 20.25 & 20.54 & - & - \\
$\rho$ & 0.5296 & 0.5750 & - & 0.5272 & 0.5257 \\
\hline
\end{tabular}

Table 2 : Results for the second series of data. 
12

\begin{tabular}{|c|c|c|c|c|c|}
\hline & True & Empirical & Univariate & \multicolumn{2}{|c|}{ EM algorithm } \\
& values & estimators & max likelihood & EM1 & EM2 \\
\hline$a_{1}$ & 0.0330 & 0.0297 & 0.0340 & 0.0343 & - \\
$b_{1}$ & 13.5 & 11.71 & 13.43 & - & - \\
$a_{2}$ & 0.0350 & 0.0340 & 0.0385 & 0.0389 & - \\
$b_{2}$ & 20 & 18.79 & 21.28 & - & - \\
$\rho$ & 0.5296 & 0.5645 & - & 0.5060 & 0.5027 \\
\hline
\end{tabular}

Table 3 : Results for the third series of data.

Figures 2(a), 2(b) and 2(c) show the evolution of $a_{i}^{(k)}$ and $\rho^{(k)}$ along the different steps of the EM algorithm (case EM1 in Table 2). We notice that the parameters $a_{i}^{(k)}$ stabilize more quickly than the parameter $\rho^{(k)}$. In other ways, the parameters $\alpha_{i}^{(k)}+\alpha_{3}^{(k)}$ for $i=1$, 2 are quickly stable (about 5 iterations), but values of $\alpha_{i}^{(k)}$ are much longer to stabilize (between 20 and 30 iterations).

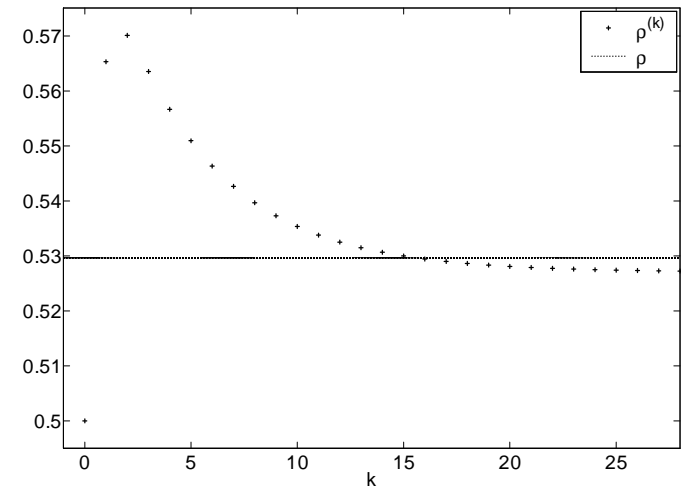

(a) Evolution of $\rho^{(k)}$



(b) Evolution of $a_{1}^{(k)}$

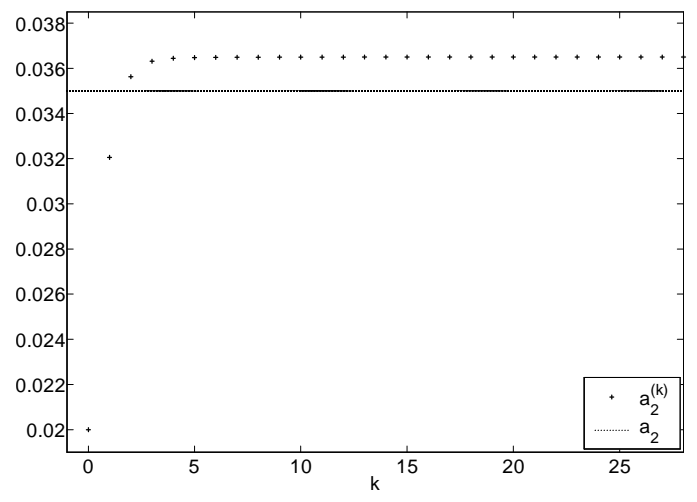

(c) Evolution of $a_{2}^{(k)}$

Figure 2. Evolution of the parameters during the execution of the EM algorithm. 
The conclusion of this section is that the estimation of the parameters $\left(a_{i}, b_{i}\right)$ by empirical and maximum likelihood methods both give satisfactory results, with a slight preference to maximum likelihood estimation. Empirical estimators of $\rho$ have a good order of magnitude, but are sometimes not precise enough. Estimators of $\rho$ obtained by EM are always reasonable. The estimation of the three parameters $\left(\alpha_{1}, \alpha_{2}, \alpha_{3}\right)$ (column EM1) seems to give slightly better results than those obtained for $\alpha_{3}$ alone (column EM2). The results obtained by the EM algorithm for parameters $a_{i}$ (column EM1) are good, with a quality quite similar to those obtained by univariate maximum likelihood estimation. Finally, the EM algorithm does not seem very sensitive to initial values, at least if the initial value of $\alpha_{3}$ is not too small.

\section{Intervention planning}

A bivariate Gamma process $X_{t}=\left(X_{t}^{(1)}, X_{t}^{(2)}\right)$ is now used to model the development of two deterioration indicators of a system. We assume that there exist thresholds $s_{i}$ $(i=1,2)$ for each indicator, above which the system is considered to be deteriorated. The system is not continuously monitored but only inspected at time intervals, with a perfect observation of the deterioration level. When one (or both) indicator(s) is (are) observed to be beyond its threshold, an intervention is undertaken. When both indicators are observed to be below their thresholds, no action is undertaken and a new inspection is planned. The time to next inspection $(\tau)$ must ensure with a high probability that neither $X_{t}^{(1)}$ nor $X_{t}^{(2)}$ go beyond their thresholds $s_{i}$ before the next inspection.

Let $\left(x_{1}, x_{2}\right) \in\left[0, s_{1}\left[\times\left[0, s_{2}[\right.\right.\right.$ be the observed deterioration level at some inspection time, say at time $t=0$ with no restriction. Also, let $\varepsilon \in] 0,1[$ be some confidence level.

Using the bivariate Gamma process, the time to the next maintenance action $\tau^{B}$ is chosen as the maximal value which ensures that the process stays below the thresholds with a probability greater than $1-\varepsilon$ :

$$
\tau^{B}=\max \left(\tau \geq 0 \text { such that } \mathbb{P}_{\left(x_{1}, x_{2}\right)}\left(X_{\tau}^{(1)}<s_{1}, X_{\tau}^{(2)}<s_{2}\right) \geq 1-\varepsilon\right)
$$

where $\mathbb{P}_{\left(x_{1}, x_{2}\right)}$ stands for the conditional probability given $\left(X_{0}^{(1)}, X_{0}^{(2)}\right)=\left(x_{1}, x_{2}\right)$. 
We have

$$
\begin{aligned}
\mathbb{P}_{\left(x_{1}, x_{2}\right)}\left(X_{t}^{(1)}<s_{1}, X_{t}^{(2)}<s_{2}\right) & =\mathbb{P}_{(0,0)}\left(X_{t}^{(1)}<s_{1}-x_{1}, X_{t}^{(2)}<s_{2}-x_{2}\right) \\
& =\int_{0}^{s_{1}-x_{1}} \int_{0}^{s_{2}-x_{2}} g_{t}\left(y_{1}, y_{2}\right) d y_{1} d y_{2}
\end{aligned}
$$

where $g_{t}$ is the p.d.f. of $X_{t}$ (see Eq. (2)). This quantity is continuous and strictly decreasing in $t$. If we set Eq. (8) equal to $1-\varepsilon$, the time $\tau^{B}$ may hence be numerically computed as the unique solution to this equation in $t$.

Without a bivariate model, a natural time to next maintenance action for the system is:

$$
\begin{aligned}
\tau^{U} & =\max \left(\tau \geq 0 \text { such that } \mathbb{P}_{x_{1}}\left(X_{\tau}^{(1)}<s_{1}\right) \geq 1-\varepsilon \text { and } \mathbb{P}_{x_{2}}\left(X_{\tau}^{(2)}<s_{2}\right) \geq 1-\varepsilon\right) \\
& =\min \left(\tau^{(1)}, \tau^{(2)}\right)
\end{aligned}
$$

with

$$
\tau^{(i)}=\max \left(\tau \geq 0 \text { such that } \mathbb{P}_{x_{i}}\left(X_{\tau}^{(i)}<s_{i}\right) \geq 1-\varepsilon\right)
$$

where $\mathbb{P}_{x_{i}}$ stands for the conditional probability given $X_{0}^{(i)}=x_{i}$.

Using

$$
\mathbb{P}_{x_{i}}\left(X_{t}^{(i)}<s_{i}\right)=\mathbb{P}_{0}\left(X_{t}^{(i)}<s_{i}-x_{i}\right)=F_{a_{i} t, b_{i}}\left(s_{i}-x_{i}\right)
$$

where $F_{a_{i} t, b_{i}}(x)$ is the cumulative distribution function of the distribution $\Gamma\left(a_{i} t, b_{i}\right)$, the time $\tau^{(i)}$ is computed by solving the equation $F_{a_{i} \tau^{(i)}, b_{i}}\left(s_{i}-x_{i}\right)=1-\varepsilon$, for $i=1,2$, and $\tau^{U}=\min \left(\tau^{(1)}, \tau^{(2)}\right)$ is derived.

Clearly we have :

$$
\begin{aligned}
1-\varepsilon & =\mathbb{P}_{\left(x_{1}, x_{2}\right)}\left(X_{\tau^{B}}^{(1)}<s_{1}, X_{\tau^{B}}^{(2)}<s_{2}\right) \\
& \leq \min \left(\mathbb{P}_{x_{1}}\left(X_{\tau^{B}}^{(1)}<s_{1}\right), \mathbb{P}_{x_{2}}\left(X_{\tau^{B}}^{(2)}<s_{2}\right)\right),
\end{aligned}
$$

which leads to $\tau^{B} \leq \min \left(\tau^{(1)}, \tau^{(2)}\right)=\tau^{U}$.

The goal now is to study the difference between $\tau^{U}$ and $\tau^{B}$, and more generally, to understand the influence of the dependence between both components of $X_{t}$ on $\tau^{B}$. We first provide numerical examples. 
Taking $a_{1}=0.03, b_{1}=20, \epsilon=0.5$ and $s_{1}=s_{2}=1$, and different values for $a_{2}, b_{2}$, $x_{1}$ and $x_{2}$, Table 4 gives the corresponding values for $\rho_{\max }$ (as provided by (1)) and the resulting $\tau^{(1)}, \tau^{(2)}, \tau^{U}$ and $\tau^{B}\left(\rho_{\max }\right)$.

\begin{tabular}{|c|c|c|c|c|c|c|c|c|c|c|}
\hline & $a_{1}$ & $a_{2}$ & $b_{1}$ & $b_{2}$ & $x_{1}$ & $x_{2}$ & $\rho_{\max }$ & $\tau^{(1)}$ & $\tau^{(2)}$ & $\tau^{U}$ \\
\hline case 1 & 0.03 & 0.03 & 20 & 20 & 0.2 & 0.2 & 1 & 341.12 & 341.12 & 341.12 \\
\hline case 2 & 0.03 & 0.03 & 20 & 20 & 0.2 & 0.5 & 1 & 187.32 & 341.12 & 187.32 \\
\hline case 3 & 0.03 & 0.04 & 20 & 20 & 0.2 & 0.2 & 0.866 & 341.12 & 255.84 & 255.84 \\
\hline case 4 & 0.03 & 0.04 & 20 & 20 & 0.4 & 0.2 & 0.866 & 237.33 & 255.84 & 237.33 \\
\hline
\end{tabular}

Table 4 : Four different combinations of values for $a_{2}, b_{2}, x_{1}$ and $x_{2}$, and the resulting $\rho_{\max }, \tau^{(1)}, \tau^{(2)}, \tau^{U}$ and $\tau^{B}\left(\rho_{\max }\right)$.

The value of $\tau^{B}$ is plotted with respect to $\rho$ in the Figures 3 for the different cases of Table 4, and the corresponding value of $\tau^{U}$ is indicated.

In these figures, one can observe that both $\tau^{B}=\tau^{U}$ and $\tau^{B}<\tau^{U}$ are possible. One can also observe some monotony property of $\tau_{B}$ with respect of the dependence between $X_{t}^{(1)}$ and $X_{t}^{(2)}$. More specifically, we have the following result:

Proposition 4.1: With all other parameters fixed, the bivariate preventive time $\tau^{B}$ is an increasing function of the linear correlation coefficient $\rho$.

Proof: Let us consider two bivariate Gamma processes $X_{t}$ and $\tilde{X}_{t}$ with the same marginal processes and linear correlation coefficients such that $\rho \leq \tilde{\rho}$. Let us denote by $U(x)$ and $\tilde{U}(x)$ their respective tail integral functions provided by $(3)$. We have $U(x) \leq \tilde{U}(x)$. It is proved in (Bäuerle et al. 2008) (theorem 4.8 and lemma 4.9) that if $U(x) \leq \tilde{U}(x)$ for all $x$ in $\mathbb{R}_{+}^{2}$, then $X \leq \tilde{X}$ with respect to the concordance order. This implies that

$$
\mathbb{P}_{x_{1}, x_{2}}\left(X_{t}^{(1)}<s_{1}, X_{t}^{(2)}<s_{2}\right) \leq \mathbb{P}_{x_{1}, x_{2}}\left(\tilde{X}_{t}^{(1)}<s_{1}, \tilde{X}_{t}^{(2)}<s_{2}\right)
$$

for all $t, x_{1} \leq s_{1}$ and $x_{2} \leq s_{2}$. Then $\tau^{B} \leq \tilde{\tau}^{B}$.

In conclusion to this section, one can see that using a bivariate model instead of two separate univariate models generally shortens the time to the next maintenance action $\left(\tau^{B} \leq \tau^{U}\right)$. If both processes are correlated, the frequency of inspections must then be increased in order to achieve the same safety level as in the uncorrelated case. So, if 


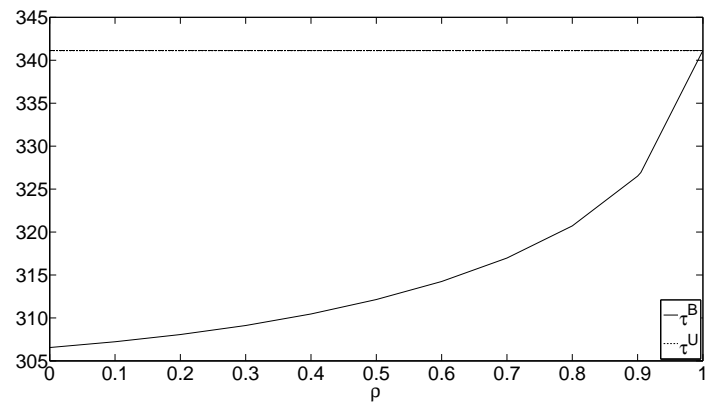

(a) Case 1



(c) Case 3

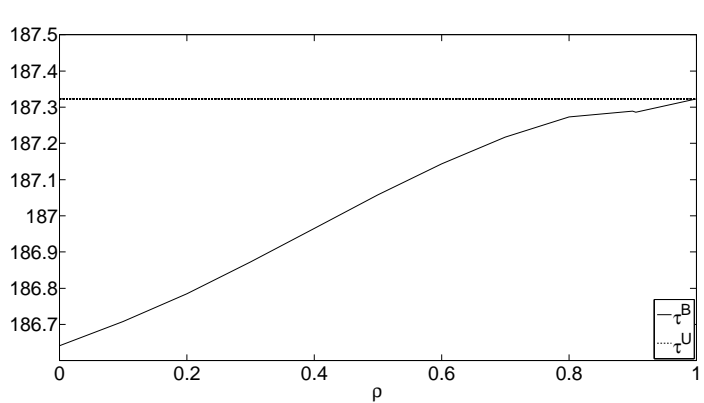

(b) Case 2

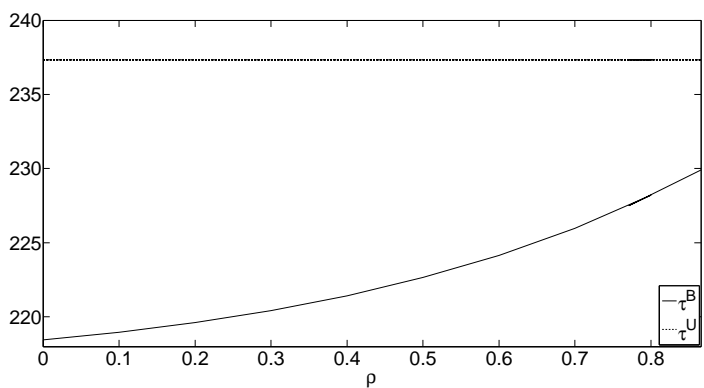

(d) Case 4

Figure 3. $\tau^{B}$ with respect to $\rho$ and $\tau^{U}$, for the four cases of Table 4

dependence is ignored, the policy is not as safe as it should be. Also, the optimal time to the next preventive maintenance action is increasing with dependence $\left(\tau^{B}\right.$ increases with $\rho$ ), which implies that the error made when considering separate models $\left(\tau^{U}\right)$ is all the more important that the components are less dependent. This also implies that the safest attitude, in case of an unknown correlation, is to consider both components as independent and chose $\tau=\tau^{\perp}$, where

$$
\tau^{\perp}=\max \left(\tau \geq 0 \text { such that } \mathbb{P}_{x_{1}}\left(X_{\tau}^{(1)}<s_{1}\right) \mathbb{P}_{x_{2}}\left(X_{\tau}^{(2)}<s_{2}\right) \geq 1-\varepsilon\right)
$$

\section{Application to track maintenance}

A bivariate Gamma process is now used to model the development of the two track indicators NL and NT (see the Introduction) and times to next intervention are computed, as described in the previous section.

Using univariate maximum likelihood and EM methods on data corresponding to 
the Paris-Lyon high-speed line provides the estimations $\hat{a}_{1}=0.0355, \hat{b}_{1}=19.19, \hat{a}_{2}=$ $0.0387, \hat{b}_{2}=29.72, \hat{\rho}=0.5262$. Usual thresholds are $s_{1}=0.9$ for NL and $s_{2}=0.75$ for NT. With these values, $\tau^{(1)}, \tau^{(2)}$ and $\tau^{B}$ are plotted in Figure 4 with respect of $x_{2}$ when $x_{1}$ is fixed $\left(x_{1}=0.4\right)$. In that case $\tau^{(1)}=150$.

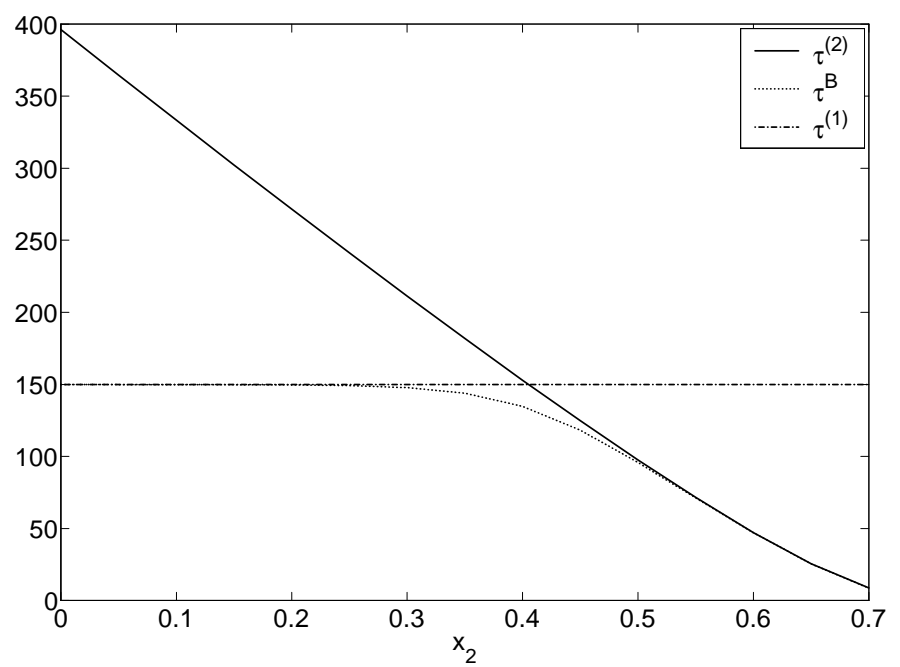

Figure 4. $\tau^{(1)}, \tau^{(2)}$ and $\tau^{B}$ with respect to $x_{2}$ with $x_{1}=0.4$

Figure 4 shows that taking into account the single information $x_{1}=0.4$ as presently done at the SNCF may lead to too late maintenance actions. As an example, if $x_{2}=0.4$, one has $\tau^{B}=134.7$ (and $\tau^{(2)}=152.9$ ). The preventive maintenance action based only on NL is consequently scheduled 15 days too late. If $x_{2}=0.5$, one obtains $\tau^{B}=95.9$ $\left(\tau^{(2)}=97.5\right)$ and the maintenance action is undertaken 54 days too late. If $x_{2}=0.6$, one obtains $\tau^{B}=47.1\left(\tau^{(2)}=47.2\right)$ and this is 103 days too late.

Concluding this section, one can finally observe that if $x_{1}$ is not too close to $x_{2}$, the value $\tau^{U}=\min \left(\tau^{(1)}, \tau^{(2)}\right)$ seams reasonable for maintenance scheduling (see Figure 4), contrary to the currently used $\tau^{(1)}$, which may entail large delays in its planning (more than 100 days in our example). If $x_{1}$ is close to $x_{2}$, the values of $\tau^{U}$ and $\tau^{B}$ have the same order of magnitude, with $\tau^{U}>\tau^{B}$ however, so that the preventive maintenance action is again planned too lately (15 days in the example). 


\section{Conclusion}

A bivariate Gamma process has been used to model the development of two deterioration indicators. Different estimation methods have been proposed for the parameters and tested on simulated data. Based on these tests, the best estimators seem provided by univariate likelihood maximization for the marginal parameters and by an EM algorithm for the correlation coefficient.

Preventive maintenance scheduling has then been studied for a system that deteriorates according to a bivariate Gamma process. In particular, it has been shown that, given an observed bivariate deterioration level, the optimal time to maintenance is increasing with dependence. It has been proven that the optimal time to maintenance is always shorter when taking into account the dependence between the two deterioration indicators than when considering them separately (or only considering one of them).

Finally, a bivariate Gamma process has been used to study a real track maintenance problem. The application shows that when both observed deterioration indicators are close to each other, the bivariate process gives safer results for maintenance scheduling than both univariate processes considered separately or one single univariate process, with the same order of magnitude in each case however. When the observed deterioration indicators are clearly different, considering one single univariate process as it is done in current track maintenance, may lead to clearly inadaquate results. The preventive maintenance action is scheduled too late and the track passes to a deteriorated state with a high probability. This application to real data of railway track deterioration hence shows the interest of a bivariate model for a correct definition of a maintenance strategy.

\section{References}

Abdel-Hameed, M., 1975. A gamma wear process, IEEE Transactions on Reliability, 24 (2), 152-153.

Bäuerle, N., Blatter, A. and Müller, A., 2008. Dependence properties and comparison results for Lévy processes, Mathematical Methods of Operational Research, 67, 161-186.

Buijs, F.A., Hall, J.W., van Noortwijk, J.M. and Sayers, P.B., 2005. Time-dependent reliability analysis of flood defences using gamma processes, in G. Augusti, G.I. Schuëller, and M. Ciampoli, editors, Safety and Reliability of Engineering Systems and Struc- 
tures; Proceedings of the Nin'th International Conference on Structural Safety and Reliability (ICOSSAR), Rome, Italy, 19-23 June 2005, 2209-2216. Rotterdam: Millpress.

Cinlar, E., Z.P. Bazant, Z.P. and Osman, E., 1977. Stochastic process for extrapolating concrete creep, Journal of the Engineering Mechanics Division, 103 (EM6), 1069-1088.

Dempster, A.P., Laird, N.M. and Rubin, D.B., 1977. Maximum likelihood from incomplete data via the EM algorithm (with discussion), Journal of the Royal Statistical Society, Series B, 39, 1-38.

Devroye, L., 1986. Non-Uniform Random Variate Generation. Springer.

Dufresne, F., Gerber, H.U. and Shin, E.S.W., 2000. Risk theory with the gamma process, Astin Bulletin, 21, 177-192.

Grall, A., Dieulle, L., Berenguer, C. and Roussignol, M., 2002. Continuous - time predictive - maintenance scheduling for a deteriorating system, IEEE Transactions on Reliability, 51, 141-150.

Joshi, M. S., Stacey, A.M., 2006. Intensity Gamma : a new approach to pricing portfolio credit derivatives, Risk magazine, 6 .

Kallsen, J. and Tankov, P., 2006. Characterization of dependence of multidimensional Lévy processes using Lévy copulas, Journal of Multivariate Analysis, 97, 1551-1572.

Meier-Hirmer, C., Riboulet, G., Sourget, F. and Roussignol, M., 2009. Maintenance optimisation for system with a Gamma deterioration process and intervention delay: application to track maintenance, Journal of Risk and Reliability, 223, 3, 189-198.

Newby, M. and Baker, C.T., 2006. A bivariate process model for maintenance and inspection planning, International Journal of Pressure Vessels ans Piping, 83, 270-275.

Nicolai, R.P., Dekker, R. and van Noortwijk, J.M., 2007. A comparison of models for measurable deterioration: An application to coatings on stell structures, Reliability Engineering and System Safety, 92, 1635-1650.

van Noortwijk, J.M., 2009. A survey of the application of gamma processes in maintenance, Reliability Engineering 85 System Safety, 94 (1), 2-21.

van Noortwijk, J.M. and Pandey, M.D., 2004. A stochastic deterioration process for time-dependent reliability analysis, In M.A. Maes and L. Huyse, editors, Proceedings of the Eleventh IFIP WG 7.5 Working Conference on Reliability and Optimization of Structural Systems, 2-5 November 2003, Banff, Canada, 259-265, London: Taylor \& Francis Group. 
Zuckerman, D., 1978. Optimal replacement policy for the case where the damage process is a one-sided Lévy process, Stochastic Processes and their Applications, 7, 141-151. 